TRANSACTIONS OF THE

AMERICAN MATHEMATICAL SOCIETY

Volume 358, Number 2, Pages 703-726

S 0002-9947(05)03751-7

Article electronically published on March 10, 2005

\title{
ON THE ANDREWS-STANLEY REFINEMENT OF RAMANUJAN'S PARTITION CONGRUENCE MODULO 5 AND GENERALIZATIONS
}

\author{
ALEXANDER BERKOVICH AND FRANK G. GARVAN
}

AbStRact. In a recent study of sign-balanced, labelled posets, Stanley introduced a new integral partition statistic

$$
\operatorname{srank}(\pi)=\mathcal{O}(\pi)-\mathcal{O}\left(\pi^{\prime}\right),
$$

where $\mathcal{O}(\pi)$ denotes the number of odd parts of the partition $\pi$ and $\pi^{\prime}$ is the conjugate of $\pi$. In a forthcoming paper, Andrews proved the following refinement of Ramanujan's partition congruence mod 5:

$$
\begin{aligned}
p_{0}(5 n+4) & \equiv p_{2}(5 n+4) \equiv 0 \quad(\bmod 5), \\
p(n) & =p_{0}(n)+p_{2}(n),
\end{aligned}
$$

where $p_{i}(n)(i=0,2)$ denotes the number of partitions of $n$ with srank $\equiv i$ $(\bmod 4)$ and $p(n)$ is the number of unrestricted partitions of $n$. Andrews asked for a partition statistic that would divide the partitions enumerated by $p_{i}(5 n+4)(i=0,2)$ into five equinumerous classes.

In this paper we discuss three such statistics: the ST-crank, the 2-quotientrank and the 5-core-crank. The first one, while new, is intimately related to the Andrews-Garvan (1988) crank. The second one is in terms of the 2-quotient of a partition. The third one was introduced by Garvan, Kim and Stanton in 1990. We use it in our combinatorial proof of the Andrews refinement. Remarkably, the Andrews result is a simple consequence of a stronger refinement of Ramanujan's congruence mod 5. This more general refinement uses a new partition statistic which we term the BG-rank. We employ the BG-rank to prove new partition congruences modulo 5. Finally, we discuss some new formulas for partitions that are 5-cores and discuss an intriguing relation between 3-cores and the Andrews-Garvan crank.

\section{INTRODUCTION}

Let $p(n)$ be the number of unrestricted partitions of $n$. Ramanujan discovered and later proved that

$$
\begin{aligned}
p(5 n+4) \equiv 0 & (\bmod 5), \\
p(7 n+5) \equiv 0 & (\bmod 7), \\
p(11 n+6) & \equiv 0 \quad(\bmod 11) .
\end{aligned}
$$

Dyson [5] was the first to consider combinatorial explanations of these congruences. He defined the rank of a partition as the largest part minus the number of parts and

Received by the editors January 12, 2004 and, in revised form, February 24, 2004.

2000 Mathematics Subject Classification. Primary 11P81, 11P83; Secondary 05A17, 05A19.

Key words and phrases. Partitions, t-cores, ranks, cranks, Stanley's statistic, Ramanujan's congruences. 
made the empirical observations that

$$
\begin{array}{ll}
N(k, 5,5 n+4)=\frac{p(5 n+4)}{5}, & 0 \leq k \leq 4, \\
N(k, 7,7 n+5)=\frac{p(7 n+5)}{7}, & 0 \leq k \leq 6,
\end{array}
$$

where $N(k, m, n)$ denotes the number of partitions of $n$ with rank congruent to $k$ modulo $m$. Equation (1.4) means that the residue of the rank mod 5 divides the partitions of $5 n+4$ into five equal classes. Similarly, (1.5) implies that the residue of the rank mod 7 divides the partitions of $7 n+5$ into seven equal classes. Dyson's rank failed to explain (1.3), and so Dyson conjectured the existence of a hypothetical statistic, called the crank, that would explain the Ramanujan congruence mod 11. Identities (1.4)-(1.5) were later proved by Atkin and Swinnerton-Dyer [3]. Andrews and Garvan 2] found a crank for all three Ramanujan congruences (1.1)-(1.3). Their crank is defined as follows:

$$
\operatorname{crank}(\pi)= \begin{cases}\ell(\pi), & \text { if } \mu(\pi)=0, \\ \widetilde{\nu}(\pi)-\mu(\pi), & \text { if } \mu(\pi)>0,\end{cases}
$$

where $\ell(\pi)$ denotes the largest part of $\pi, \mu(\pi)$ denotes the number of ones in $\pi$ and $\widetilde{\nu}(\pi)$ denotes the number of parts of $\pi$ larger than $\mu(\pi)$.

Later, Garvan, Kim and Stanton [9] found different cranks, which also explained all three congruences (1.1)-(1.3). Their approach made essential use of $t$-cores of partitions and led to explicit bijections between various equinumerous classes. In particular, they provided what amounts to a combinatorial proof of the formula

$$
\sum_{n \geq 0} p(5 n+4) q^{n}=5 \prod_{m \geq 1} \frac{\left(1-q^{5 m}\right)^{5}}{\left(1-q^{m}\right)^{6}},
$$

considered by Hardy to be an example of Ramanujan's best work.

The main results of 2] can be summarized as

$$
\begin{array}{cl}
M(k, 5,5 n+4)=\frac{p(5 n+4)}{5}, & 0 \leq k \leq 4, \\
M(k, 7,7 n+5)=\frac{p(7 n+5)}{7}, & 0 \leq k \leq 6, \\
M(k, 11,11 n+6)=\frac{p(11 n+6)}{11}, & 0 \leq k \leq 10,
\end{array}
$$

and

$$
\begin{gathered}
1+\left(x+x^{-1}-1\right) q+\sum_{n>1} \sum_{m} \tilde{M}(m, n) x^{m} q^{n} \\
=\prod_{n \geq 1} \frac{\left(1-q^{n}\right)}{\left(1-x q^{n}\right)\left(1-x^{-1} q^{n}\right)},
\end{gathered}
$$

where $\tilde{M}(m, n)$ denotes the number of partitions of $n$ with crank $m$ and $M(k, m, n)$ denotes the number of partitions of $n$ with crank congruent to $k$ modulo $m$.

In 7. Garvan found a refinement of (1.1)

$$
M(k, 2,5 n+4) \equiv 0 \quad(\bmod 5), \quad k=0,1,
$$


together with the combinatorial interpretation

$$
M(2 k+\alpha, 10,5 n+4)=\frac{M(\alpha, 2,5 n+4)}{5}, \quad 0 \leq k \leq 4,
$$

with $\alpha=0,1$.

Recently, a very different refinement of (1.1) was given by Andrews [1]. Building on the work of Stanley [14, Andrews examined partitions $\pi$ classified according to $\mathcal{O}(\pi)$ and $\mathcal{O}\left(\pi^{\prime}\right)$, where $\mathcal{O}(\pi)$ denotes the number of odd parts of the partition $\pi$ and $\pi^{\prime}$ is the conjugate of $\pi$. He used recursive relations to show that

$$
G(z, y, q):=\sum_{n, r, s \geq 0} S(n, r, s) q^{n} z^{r} y^{s}=\frac{\left(-z y q ; q^{2}\right)_{\infty}}{\left(q^{4} ; q^{4}\right)_{\infty}\left(z^{2} q^{2} ; q^{4}\right)_{\infty}\left(y^{2} q^{2} ; q^{4}\right)_{\infty}}
$$

where $S(n, r, s)$ denotes the number of partitions $\pi$ of $n$ with $\mathcal{O}(\pi)=r, \mathcal{O}\left(\pi^{\prime}\right)=s$, and

$$
\begin{gathered}
(a ; q)_{\infty}=\lim _{n \rightarrow \infty}(a ; q)_{n}, \\
(a ; q)_{n}=(a)_{n}= \begin{cases}1, & \text { if } n=0 \\
\prod_{j=0}^{n-1}\left(1-a q^{j}\right), & \text { if } n>0 .\end{cases}
\end{gathered}
$$

A direct combinatorial proof of (1.14) was later given by A. Sills [13, A. J. Yee 15] and C. Boulet 4]. Actually, C. Boulet proved a stronger version of (1.14) with one extra parameter. We define the Stanley rank of a partition $\pi$ as

$$
\operatorname{srank}(\pi)=\mathcal{O}(\pi)-\mathcal{O}\left(\pi^{\prime}\right) .
$$

It is easy to see that

$$
\operatorname{srank}(\pi) \equiv 0 \quad(\bmod 2),
$$

so that

$$
p(n)=p_{0}(n)+p_{2}(n),
$$

where $p_{i}(n)(i=0,2)$ denotes the number of partitions of $n$ with srank $\equiv i(\bmod 4)$. We note that (1.14) with $z=y^{-1}=\sqrt{-1}$ immediately implies the Stanley formula [14, p. 8]

$$
\sum_{n \geq 0}\left(p_{0}(n)-p_{2}(n)\right) q^{n}=\frac{\left(-q ; q^{2}\right)_{\infty}}{\left(q^{4} ; q^{4}\right)_{\infty}\left(-q^{2} ; q^{4}\right)_{\infty}^{2}} .
$$

Using (1.1), (1.19) and (1.20), Andrews proved the following refinement of (1.1):

$$
p_{0}(5 n+4) \equiv p_{2}(5 n+4) \equiv 0 \quad(\bmod 5) .
$$

His proof of (1.21) was analytic and so at the end of 1 he posed the problem of finding a partition statistic that would give a combinatorial interpretation of (1.21). The first goal of this paper is to provide such an interpretation. It turns out that there are several distinct integral partition statistics, whose residue mod 5 split the partitions enumerated by $p_{i}(5 n+4)$ (with $\left.i=0,2\right)$ into five equal classes. The first statistic, which we call the St-crank, is new. However, it is intimately related to the Andrews-Garvan crank (1.6). The second statistic, which we call the 2-quotient-rank, is also new. Unexpectedly, the third statistic is the 5-corecrank, introduced by Garvan, Kim and Stanton [9]. This statistic not only provides the desired combinatorial interpretation, but it also leads to a direct combinatorial proof of (1.21). 
Our second goal here is to show that Andrews' result (1.21) is a straightforward corollary of the new refinement of (1.1). This stronger refinement uses a new partition statistic, which we term the BG-rank. Remarkably, the BG-rank enables us to discover and prove new partition congruences mod 5.

The rest of this paper is organized as follows. In Section 2 we define the St-crank and show that it is indeed a statistic asked for in [1. In Section 3 we give another combinatorial interpretation of (1.21), discuss a surprising relation between 3-cores and the Andrews-Garvan crank, and then briefly review the development in [9]. In Section 4 we establish a number of new formulas for partitions that are 5-cores and outline a combinatorial proof of (1.21). The hardest parts of this proof are dealt with in Sections 5 and 6. Finally, in Section 7 we introduce the BG-rank and use it to prove new partition congruences $\bmod 5$.

\section{THE ST-CRANK}

We begin with some preliminaries about partitions and their conjugates. A partition $\pi$ is a nonincreasing sequence

$$
\pi=\left(\lambda_{1}, \lambda_{2}, \lambda_{3}, \ldots\right)
$$

of nonnegative integers (parts)

$$
\lambda_{1} \geq \lambda_{2} \geq \lambda_{3} \geq \cdots .
$$

The weight of $\pi$, denoted by $|\pi|$, is the sum of parts

$$
|\pi|=\lambda_{1}+\lambda_{2}+\lambda_{3}+\cdots \text {. }
$$

If $|\pi|=n$, then we say that $\pi$ is a partition of $n$. Often it is convenient to use another notation for $\pi$

$$
\pi=\left(1^{f_{1}}, 2^{f_{2}}, 3^{f_{3}}, \ldots\right),
$$

which indicates the number of times each integer occurs as a part. The number $f_{i}=f_{i}(\pi)$ is called the frequency of $i$ in $\pi$. The conjugate of $\pi$ is the partition $\pi^{\prime}=\left(\lambda_{1}^{\prime}, \lambda_{2}^{\prime}, \lambda_{3}^{\prime}, \ldots\right)$ with

$$
\begin{aligned}
& \lambda_{1}^{\prime}=f_{1}+f_{2}+f_{3}+f_{4}+\cdots \\
& \lambda_{2}^{\prime}=f_{2}+f_{3}+f_{4}+\cdots \\
& \lambda_{3}^{\prime}=f_{3}+f_{4}+\cdots
\end{aligned}
$$

Next, we discuss two bijections. The first one relates $\pi$ and bipartitions $\left(\pi_{1}, \pi_{2}\right)$, where $\pi_{2}$ is a partition with no repeated even parts.

\section{Bijection 1.}

$$
\pi \stackrel{1}{\longrightarrow}\left(\pi_{1}, \pi_{2}\right)
$$

where

$$
\begin{aligned}
\pi & =\left(1^{f_{1}}, 2^{f_{2}}, 3^{f_{3}}, \ldots\right), \\
\pi_{1} & =\left(1^{\left\lfloor f_{2} / 2\right\rfloor}, 2^{\left\lfloor f_{4} / 2\right\rfloor}, 3^{\left\lfloor f_{6} / 2\right\rfloor}, \ldots\right), \\
\pi_{2} & =\left(1^{f_{1}}, 2^{\left\{f_{2}\right\}}, 3^{f_{3}}, 4^{\left\{f_{2}\right\}}, \ldots\right),
\end{aligned}
$$


$\lfloor x\rfloor$ is the largest integer $\leq x$, and

$$
\{x\}=x-2\lfloor x / 2\rfloor .
$$

Indeed, remove from $\pi$ the maximum even number of even parts. The resulting partition is $\pi_{2}$. The removed even parts can be organized into a new partition $\left(2^{2\left\lfloor f_{2} / 2\right\rfloor}, 4^{2\left\lfloor f_{4} / 2\right\rfloor}, 6^{2\left\lfloor f_{6} / 2\right\rfloor}, \ldots\right)$, which can easily be mapped onto $\pi_{1}$. Clearly, we have

$$
\begin{aligned}
|\pi| & =4\left|\pi_{1}\right|+\left|\pi_{2}\right|, \\
\operatorname{srank}(\pi) & =\operatorname{srank}\left(\pi_{2}\right),
\end{aligned}
$$

so that

$$
\begin{aligned}
\sum_{\pi} q^{|\pi|} y^{\operatorname{srank}(\pi)} & =\sum_{\pi_{1}} q^{4\left|\pi_{1}\right|} \sum_{\pi_{2}} q^{\left|\pi_{2}\right|} y^{\operatorname{srank}\left(\pi_{2}\right)} \\
& =\frac{1}{\left(q^{4} ; q^{4}\right)_{\infty}} \sum_{\pi_{2}} q^{\left|\pi_{2}\right|} y^{\operatorname{srank}\left(\pi_{2}\right)} .
\end{aligned}
$$

Comparing (2.8) and (1.14) with $z y=1$, we see that

$$
\sum_{\pi_{2}} q^{\left|\pi_{2}\right|} y^{\operatorname{srank}\left(\pi_{2}\right)}=\frac{\left(-q ; q^{2}\right)_{\infty}}{\left(y^{2} q^{2} ; q^{4}\right)_{\infty}\left(q^{2} / y^{2} ; q^{4}\right)_{\infty}}
$$

where the sum is over all partitions with no repeated even parts.

To describe our second bijection we require a few definitions. We say that $\pi_{A}$ is a partition of type $\mathrm{A}$ iff $\pi_{A} \stackrel{1}{\longrightarrow}\left((1), \pi_{2}\right)$. We say that $\pi_{B}=\left(\lambda_{1}, \lambda_{2}, \lambda_{3}, \ldots\right)$ is a partition of type B iff either $\left|\pi_{B}\right| \neq 4, \lambda_{1}-\lambda_{2} \geq 2, \lambda_{1}^{\prime}-\lambda_{2}^{\prime} \geq 2, \lambda_{1}-2$ and $\lambda_{2}$ are not identical even integers and $\pi_{B}$ has no repeated even parts, or $\pi_{B}=(3,1)$. Obviously, $\pi_{B} \stackrel{1}{\longrightarrow}\left((0), \pi_{B}\right)$. Our second bijection relates partitions of type A and B.

\section{Bijection 2.}

$$
\pi_{A} \stackrel{2}{\longrightarrow} \pi_{B}
$$

where

$$
\begin{aligned}
& \pi_{A}=\left(1^{f_{1}}, 2^{f_{2}}, 3^{f_{3}}, \ldots, m^{f_{m}}\right), \\
& \pi_{B}=\left\{\begin{array}{cl}
\left(1^{f_{1}+2}, 2^{f_{2}-2}, 3^{f_{3}}, 4^{f_{4}}, \ldots,\right. & \\
\left.(m-1)^{f_{m-1}}, m^{f_{m}-1},(m+2)^{1}\right), & \text { if } m>2, \\
\left(1^{f_{1}+2}, 4^{1}\right), & \text { if } m=2, f_{2}=3, \\
\left(1^{f_{1}+1}, 3^{1}\right), & \text { if } m=2, f_{2}=2,
\end{array}\right.
\end{aligned}
$$

$m \geq 2, f_{2}=2,3$, and $f_{2 i}=0,1$ for $i>1$.

Clearly, we have

$$
\begin{aligned}
\left|\pi_{A}\right| & =\left|\pi_{B}\right|, \\
\operatorname{srank}\left(\pi_{A}\right) & =\operatorname{srank}\left(\pi_{B}\right) .
\end{aligned}
$$

Next, we define a new partition statistic

$$
\operatorname{St}-\operatorname{crank}(\pi)=\operatorname{crank}\left(\pi_{1}\right)+\frac{1}{2} \operatorname{srank}(\pi)+\Psi(\pi),
$$




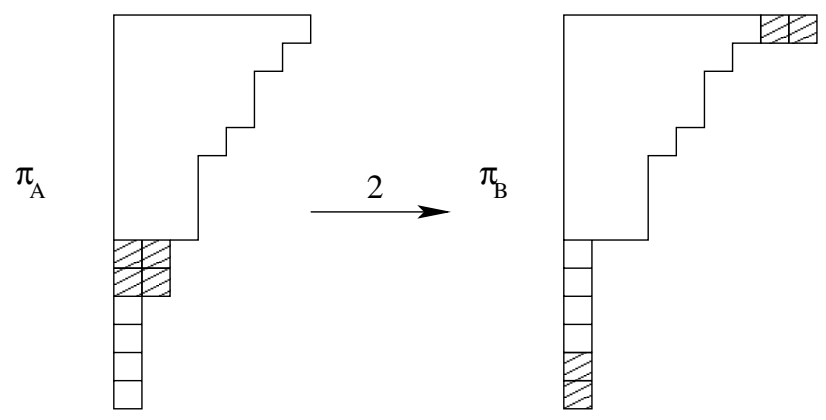

Figure 1. Graphical illustration of Bijection 2

where $\pi_{1}$ is determined by $\pi \stackrel{1}{\longrightarrow}\left(\pi_{1}, \pi_{2}\right)$, and the correction term $\Psi(\pi)=1$ if $\pi$ is of type $\mathrm{B}$ and zero, otherwise. We note that

$$
\operatorname{St}-\operatorname{crank}\left(\pi_{A}\right)=-1+\frac{1}{2} \operatorname{srank}\left(\pi_{A}\right)
$$

and

$$
\operatorname{St}-\operatorname{crank}\left(\pi_{B}\right)=1+\frac{1}{2} \operatorname{srank}\left(\pi_{B}\right) \text {. }
$$

We give some examples. Let $\pi=\left(1^{2}, 2^{4}, 3^{2}, 4^{1}, 5^{1}, 6^{2}\right)$. Then $\pi \stackrel{1}{\longrightarrow}\left(\left(1^{2}, 3^{1}\right)\right.$, $\left.\left(1^{2}, 3^{2}, 4^{1}, 5^{1}\right)\right)$ so that $\operatorname{St}-\operatorname{crank}(\pi)=(1-2)+(5-1) / 2+0=1$. Next, we consider a partition of type B. Let $\pi_{B}=\left(1^{2}, 3^{1}, 5^{1}\right)$. Then St-crank $\left(\pi_{B}\right)=0+(4-2) / 2+1=2$.

Equipped with the definitions above, we can now prove the following lemma.

Lemma 2.1. If

$$
g(x, y, q):=\sum_{\pi} q^{|\pi|} x^{\mathrm{St}-\operatorname{crank}(\pi)} y^{\operatorname{srank}(\pi)},
$$

then $g(x, y, q)$ has the product representation

$$
g(x, y, q)=\frac{\left(q^{4} ; q^{4}\right)_{\infty}\left(-q ; q^{2}\right)_{\infty}}{\left(q^{4} x, q^{4} / x, q^{2} y^{2} x, q^{2} /\left(y^{2} x\right) ; q^{4}\right)_{\infty}},
$$

where

$$
\left(a_{1}, a_{2}, a_{2}, \ldots ; q\right)_{\infty}=\left(a_{1} ; q\right)_{\infty}\left(a_{2} ; q\right)_{\infty}\left(a_{3} ; q\right)_{\infty} \cdots .
$$

Proof. If $\pi$ is not of type B and $\pi \stackrel{1}{\longrightarrow}\left(\pi_{1}, \pi_{2}\right)$, then using (2.6)-(2.7) and (2.12) we find that

$$
q^{|\pi|} x^{\operatorname{St}-\operatorname{crank}(\pi)} y^{\operatorname{srank}(\pi)}=q^{4\left|\pi_{1}\right|+\left|\pi_{2}\right|} x^{\operatorname{crank}\left(\pi_{1}\right)}\left(x y^{2}\right)^{\operatorname{srank}\left(\pi_{2}\right) / 2} .
$$

On the other hand, if $\pi=\pi_{A}$ and $\pi_{A} \stackrel{2}{\longrightarrow} \pi_{B}$, then

$$
\begin{aligned}
& q^{\left|\pi_{A}\right|} x^{\mathrm{St}-\operatorname{crank}\left(\pi_{A}\right)} y^{\operatorname{srank}\left(\pi_{A}\right)}+q^{\left|\pi_{B}\right|} x^{\mathrm{St}-\operatorname{crank}\left(\pi_{B}\right)} y^{\operatorname{srank}\left(\pi_{B}\right)} \\
& \quad=q^{\left|\pi_{A}\right|}\left(x+x^{-1}-1\right)\left(x y^{2}\right)^{\operatorname{srank}\left(\pi_{A}\right) / 2}+q^{\left|\pi_{B}\right|} x^{0}\left(x y^{2}\right)^{\operatorname{srank}\left(\pi_{B}\right) / 2} .
\end{aligned}
$$

Here we have used (2.6) $-(2.7)$ and (2.10) $-(2.14)$.

Equations (2.15) and (2.16) imply that

$$
\sum_{\pi} q^{|\pi|} x^{\operatorname{St}-\operatorname{crank}(\pi)} y^{\operatorname{srank}(\pi)}=\sum_{\pi_{1}} q^{4\left|\pi_{1}\right|} w\left(x, \pi_{1}\right) \sum_{\pi_{2}} q^{\left|\pi_{2}\right|}\left(x y^{2}\right)^{\operatorname{srank}\left(\pi_{2}\right) / 2},
$$


where

$$
w\left(x, \pi_{1}\right)= \begin{cases}x+x^{-1}-1, & \text { if } \pi_{1}=(1) \\ x^{\operatorname{crank}\left(\pi_{1}\right)}, & \text { otherwise }\end{cases}
$$

We note that in the first sum on the right side of (2.17) the summation is over unrestricted partitions $\pi_{1}$, and in the second sum the summation is over partitions $\pi_{2}$ with no repeated even parts. Finally, recalling (1.11) with $q \rightarrow q^{4}$ and (2.9) with $y^{2} \rightarrow x y^{2}$, we obtain

$$
\sum_{\pi} q^{|\pi|} x^{\operatorname{St}-\operatorname{crank}(\pi)} y^{\operatorname{srank}(\pi)}=\frac{\left(q^{4} ; q^{4}\right)_{\infty}}{\left(x q^{4}, q^{4} / x ; q^{4}\right)_{\infty}} \cdot \frac{\left(-q ; q^{2}\right)_{\infty}}{\left(x y^{2} q^{2}, q^{2} /\left(x y^{2}\right) ; q^{4}\right)_{\infty}},
$$

as desired.

Next we show that

$$
\begin{aligned}
& \text { the coefficient of } q^{5 n+4} \text { in } g(\xi, 1, q)=0, \\
& \text { the coefficient of } q^{5 n+4} \text { in } g(\xi, \sqrt{-1}, q)=0,
\end{aligned}
$$

where $\xi$ is a primitive fifth root of unity $\left(\xi^{5}=1\right)$. We use the method of [6]. We need Jacobi's triple product identity

$$
\sum_{n=-\infty}^{\infty} z^{n} q^{n^{2}}=\left(q^{2},-q z,-q / z ; q^{2}\right)_{\infty}
$$

which implies that

$$
\left(q^{4} ; q^{4}\right)_{\infty}\left(-q ; q^{2}\right)_{\infty}=\left(q^{4},-q^{3},-q ; q^{4}\right)_{\infty}=\sum_{n=-\infty}^{\infty} q^{2 n^{2}+n}=\sum_{k \geq 0} q^{T_{k}}
$$

and

$$
\left(q^{2} \xi^{2}, q^{2} / \xi^{2}, q^{2} ; q^{2}\right)_{\infty}=\frac{1}{1-\xi^{2}} \sum_{m \geq 0}(-1)^{m} q^{2 T_{m}} \xi^{-2 m}\left(1-\xi^{4 m+2}\right) .
$$

Here $T_{k}=k(k+1) / 2$. By Lemma 2.1, (2.22) and (2.23) we have

$$
\begin{gathered}
g(\xi, 1, q)=\frac{\sum_{k \geq 0} q^{T_{k}}}{\left(q^{4} \xi, q^{4} / \xi, q^{2} \xi, q^{2} / \xi ; q^{4}\right)_{\infty}}=\frac{\left(q^{2} \xi^{2}, q^{2} / \xi^{2}, q^{2} ; q^{2}\right)_{\infty}}{\left(q^{10} ; q^{10}\right)_{\infty}} \sum_{k \geq 0} q^{T_{k}} \\
=\frac{1}{1-\xi^{2}} \frac{1}{\left(q^{10} ; q^{10}\right)_{\infty}} \sum_{k, m \geq 0}(-1)^{m} q^{2 T_{m}+T_{k}} \xi^{-2 m}\left(1-\xi^{4 m+2}\right) .
\end{gathered}
$$

Note that $2 T_{m}+T_{k} \equiv 4(\bmod 5)$ iff $k \equiv m \equiv 2(\bmod 5)$, but then $1-\xi^{4 m+2}=0$. This proves (2.19). The proof of (2.20) is analogous.

Let $P_{i}(k, m, n)$ denote the number of partitions of $n$ with srank $\equiv i(\bmod 4)$ and St-crank $\equiv k(\bmod m)$. Clearly,

$$
\begin{aligned}
& \sum_{k=0}^{4} \xi^{k} \sum_{n \geq 0} P_{0}(k, 5, n) q^{n}=\frac{g(\xi, 1, q)+g(\xi, \sqrt{-1}, q)}{2} \\
& \sum_{k=0}^{4} \xi^{k} \sum_{n \geq 0} P_{2}(k, 5, n) q^{n}=\frac{g(\xi, 1, q)-g(\xi, \sqrt{-1}, q)}{2} .
\end{aligned}
$$


Combining (2.19) $-(2.20)$ and (2.25) $-(2.26)$ we find that

$$
\sum_{k=0}^{4} \xi^{k} P_{i}(k, 5,5 n+4)=0(\text { for } i=0,2)
$$

which implies that

$$
P_{i}(0,5,5 n+4)=P_{i}(1,5,5 n+4)=\cdots=P_{i}(4,5,5 n+4) .
$$

On the other hand

$$
p_{i}(5 n+4)=\sum_{k=0}^{4} P_{i}(k, 5,5 n+4)
$$

so that

$$
P_{i}(k, 5,5 n+4)=\frac{1}{5} p_{i}(5 n+4),
$$

for $i=0,2$ and $k=0,1,2,3,4$. Thus, we have proved the main result of this section.

Theorem 2.2. The residue of the partition statistic St-crank mod 5 divides the partitions enumerated by $p_{i}(5 n+4)$ with $i=0,2$ into five equinumerous classes.

We illustrate this theorem in Table 1 below for the 30 partitions of 9 . These partitions are organized into five classes with six members each. In each class the first 4 members have srank $\equiv 0(\bmod 4)$ and the remaining two members have srank $\equiv 2(\bmod 4)$.

\section{TABLE 1.}

\begin{tabular}{|c|c|c|c|c|c|}
\hline & St-crank $\equiv 0(\bmod 5)$ & $1(\bmod 5)$ & $2(\bmod 5)$ & $3(\bmod 5)$ & $4(\bmod 5)$ \\
\hline srank $\equiv 0$ & $\left(3^{3}\right)$ & $\left(1^{5}, 2^{2}\right)$ & $\left(1^{4}, 2^{1}, 3^{1}\right)$ & $\left(1^{1}, 2^{4}\right)$ & $\left(1^{9}\right)$ \\
$(\bmod 4)$ & $\left(1^{3}, 2^{1}, 4^{1}\right)$ & $\left(1^{4}, 5^{1}\right)$ & $\left(1^{3}, 3^{2}\right)$ & $\left(1^{6}, 3^{1}\right)$ & $\left(1^{2}, 2^{2}, 3^{1}\right)$ \\
& $\left(1^{1}, 3^{1}, 5^{1}\right)$ & $\left(1^{2}, 2^{1}, 5^{1}\right)$ & $\left(1^{1}, 4^{2}\right)$ & $\left(1^{1}, 2^{1}, 6^{1}\right)$ & $\left(2^{3}, 3^{1}\right)$ \\
& $\left(4^{1}, 5^{1}\right)$ & $\left(9^{1}\right)$ & $\left(2^{2}, 5^{1}\right)$ & $\left(2^{1}, 7^{1}\right)$ & $\left(1^{2}, 7^{1}\right)$ \\
\hline srank $\equiv 2$ & $\left(1^{3}, 2^{3}\right)$ & $\left(1^{1}, 2^{1}, 3^{2}\right)$ & $\left(1^{5}, 4^{1}\right)$ & $\left(1^{7}, 2^{1}\right)$ & $\left(2^{1}, 3^{1}, 4^{1}\right)$ \\
$(\bmod 4)$ & $\left(1^{3}, 6^{1}\right)$ & $\left(1^{2}, 3^{1}, 4^{1}\right)$ & $\left(1^{1}, 8^{1}\right)$ & $\left(1^{1}, 2^{2}, 4^{1}\right)$ & $\left(3^{1}, 6^{1}\right)$ \\
\hline
\end{tabular}

The analogs of (2.19) -(2.20) do not hold for $7 n+5$ when $\xi^{7}=1$ or for $11 n+6$ when $\xi^{11}=1$ so that the analog of Theorem 2.2 does not hold for Ramanujan's congruence mod 7 or mod 11 .

Finally, we note that the equation

$$
\operatorname{srank}(\pi)=-\operatorname{srank}\left(\pi^{\prime}\right)
$$

implies that a partition $\pi$ is self-conjugate only if $\operatorname{srank}(\pi)=0$. This means that the involution $\pi \longrightarrow \pi^{\prime}$ has no fixed points if $\operatorname{srank}(\pi) \equiv 2(\bmod 4)$. Hence, $2 \mid p_{2}(5 n+4)$ and by (1.21) we have the stronger congruence

$$
p_{2}(5 n+4) \equiv 0 \quad(\bmod 10) .
$$




\section{3. $t$-CORES}

3.1. Preliminaries. In this section we recall some basic facts about $t$-cores and briefly review the development in 9]. A partition $\pi$ is called a $t$-core, if it has no rim hooks of length $t$ [1]. We let $a_{t}(n)$ denote the number of partitions of $n$ which are $t$-cores. In what follows, $\pi_{t \text {-core }}$ denotes a $t$-core partition. Given the diagram of a partition $\pi$ we label a cell in the $i$-th row and $j$-th column by the least nonnegative integer congruent to $j-i(\bmod t)$. The resulting diagram is called a $t$-residue diagram [11, p. 84].

We also label cells in the infinite column 0 and in the infinite row 0 in the same way, and call the resulting diagram the extended $t$-residue diagram 9 . A region $r$ in the extended diagram is the set of cells $(i, j)$ satisfying $t(r-1) \leq j-i<t r$. A cell is called exposed if it is at the end of a row. One can construct $t$ bi-infinite words $W_{0}, W_{1}, \ldots, W_{t-1}$ of two letters $N$ (not exposed) and $E$ (exposed) as follows:

The $r$-th element of $W_{k}= \begin{cases}E, & \text { if there is an exposed cell labelled } k \text { in region } r \\ N, & \text { otherwise }\end{cases}$

Let $P$ be the set of all partitions and $P_{t \text {-core }}$ be the set of all $t$-cores. There is a well-known bijection which goes back to Littlewood [12]. $\phi_{1}: P \rightarrow P_{t \text {-core }} \times P \times$ $\cdots \times P$,

$$
\begin{aligned}
\phi_{1}(\pi) & =\left(\pi_{t \text {-core }}, \vec{\pi}_{t}\right), \\
\vec{\pi}_{t} & =\left(\widehat{\pi}_{0}, \widehat{\pi}_{1}, \widehat{\pi}_{2}, \ldots, \widehat{\pi}_{t-1}\right),
\end{aligned}
$$

such that

$$
|\pi|=\left|\pi_{t \text {-core }}\right|+t \sum_{i=0}^{t-1}\left|\widehat{\pi}_{i}\right|
$$

This bijection is described in more detail in [11, 9] and [8]. The following identity is an immediate corollary of this bijection.

$$
\frac{1}{(q)_{\infty}}=\sum_{n \geq 0} p(n) q^{n}=\frac{1}{\left(q^{t} ; q^{t}\right)_{\infty}^{t}} \sum_{n \geq 0} a_{t}(n) q^{n}
$$

It can be rewritten as

$$
\sum_{n \geq 0} a_{t}(n) q^{n}=\frac{\left(q^{t} ; q^{t}\right)_{\infty}^{t}}{(q)_{\infty}}
$$

There is another bijection $\phi_{2}$, introduced in [9]. It is for t-cores only. $\phi_{2}$ : $P_{t \text {-core }} \rightarrow\left\{\vec{n}=\left(n_{0}, n_{1}, \ldots, n_{t-1}\right): n_{i} \in \mathbb{Z}, n_{0}+\cdots+n_{t-1}=0\right\}$,

$$
\phi_{2}\left(\pi_{t \text {-core }}\right)=\vec{n}=\left(n_{0}, n_{1}, n_{2}, \ldots, n_{t-1}\right) .
$$

We call $\vec{n}$ an $n$-vector. It has the following properties:

$$
\vec{n} \in \mathbb{Z}^{t}, \quad \vec{n} \cdot \overrightarrow{1}_{t}=0,
$$

and

$$
\left|\pi_{t-\text { core }}\right|=\frac{t}{2} \sum_{i=0}^{t-1} n_{i}^{2}+\sum_{i=0}^{t-1} i n_{i}
$$


where the $t$-dimensional vector $\overrightarrow{1}_{t}$ has all components equal to 1 . The generating function identity that corresponds to this second bijection is

$$
\sum_{n \geq 0} a_{t}(n) q^{n}=\sum_{\substack{\vec{n} \in \mathbb{Z}^{t} \\ \vec{n} \cdot \overrightarrow{1}_{t}=0}} q^{\frac{t}{2}\|\vec{n}\|^{2}+\vec{b}_{t} \cdot \vec{n}}
$$

Here

$$
\|\vec{n}\|^{2}=\sum_{i=0}^{t-1} n_{i}^{2}, \quad \text { and } \quad \vec{b}_{t}=(0,1,2, \ldots, t-1) .
$$

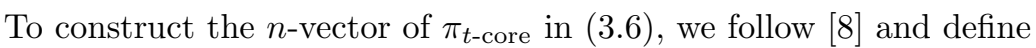

$$
\vec{r}\left(\pi_{t \text {-core }}\right)=\left(r_{0}, r_{1}, r_{2}, \ldots, r_{t-1}\right),
$$

where for $0 \leq i \leq t-1, r_{i}\left(\pi_{t \text {-core }}\right)$ denotes the number of cells labelled $i(\bmod t)$ in the $t$-residue diagram of $\pi_{t \text {-core. Then (3.6) }}$ can be given explicitly as

$$
\phi_{2}\left(\pi_{t \text {-core }}\right)=\vec{n}=\left(r_{0}-r_{1}, r_{1}-r_{2}, r_{2}-r_{3}, \ldots, r_{t-1}-r_{0}\right) .
$$

It was shown in [9] that a partition is a $t$-core with $n$-vector $\left(n_{0}, n_{1}, \ldots, n_{t-1}\right)$ iff for all $i=0, \ldots, t-1$ the bi-infinite word $W_{i}$ is of the form

$$
\begin{array}{ccccccc}
\text { Region : } & \cdots \ldots \ldots & n_{i}-1 & n_{i} & n_{i}+1 & n_{i}+2 & \ldots \ldots \ldots \\
W_{i}: & \cdots \cdots & E & E & N & N & \cdots \cdots \cdots
\end{array}
$$

We note that $\frac{t}{2}\|\vec{n}\|^{2}$ is a multiple of $t$ since $\vec{n} \cdot \overrightarrow{1}_{t}=0$. Hence by (3.4) and (3.9) we have

$$
\sum_{n \geq 0} a_{t}(t n+\delta) q^{t n+\delta}=\sum_{\substack{\vec{n} \in \mathbb{Z}^{t}, \vec{n} \cdot \overrightarrow{1}_{t}=0 \\ \vec{n} \cdot \vec{b}_{t} \equiv \delta \quad(\bmod t)}} q^{\frac{t}{2}\|\vec{n}\|^{2}+\vec{b}_{t} \cdot \vec{n}}
$$

and

$$
\sum_{n \geq 0} p(t n+\delta) q^{n}=\frac{1}{(q)_{\infty}^{t}} \sum_{n \geq 0} a_{t}(t n+\delta) q^{n}
$$

where $\delta=0,1,2, \ldots, t-1$.

3.2. The 2-quotient-rank. Having collected the necessary background on $t$-cores, we are now in a position to provide another combinatorial intrepretation of (1.21). To this end we introduce the following new partition statistic:

$$
\text { 2-quotient-rank }(\pi)=\nu\left(\widehat{\pi}_{0}\right)-\nu\left(\widehat{\pi}_{1}\right),
$$

where $\widehat{\pi}_{0}$ and $\widehat{\pi}_{1}$ are determined by

$$
\phi_{1}(\pi)=\left(\pi_{2 \text {-core }},\left(\widehat{\pi}_{0}, \widehat{\pi}_{1}\right)\right),
$$

where $\nu(\pi)$ denotes the number of parts of $\pi$. Our main result here is

Theorem 3.1. The residue of the 2-quotient-rank mod 5 divides the partitions enumerated by $p_{i}(5 n+4)$ with $i=0,2$ into five equal classes.

Proof. We start by recalling Proposition 3.1(d) in [14]:

$$
\operatorname{srank}(\pi) \equiv|\pi|-\left|\pi_{2 \text {-core }}\right| \quad(\bmod 4) .
$$

Using (3.3) with $t=2$, we obtain from (3.17)

$$
\operatorname{srank}(\pi) \equiv 2\left(\left|\widehat{\pi}_{0}\right|+\left|\widehat{\pi}_{1}\right|\right) \quad(\bmod 4) .
$$


Next, we define the generating function

$$
G_{2}(x, y, q):=\sum_{\pi} q^{|\pi|} x^{2-q u o t i e n t-\operatorname{rank}(\pi)} y^{\operatorname{srank}(\pi)} .
$$

It is possible to find a product representation for $G_{2}(x, \omega, q)$ when $\omega^{4}=1$, namely

$$
\begin{aligned}
& G_{2}(x, \omega, q)=\sum_{\substack{\pi_{2} \text {-core } \\
\pi_{0}, \pi_{1}}} q^{\left|\pi_{2-\text { core }}\right|+2\left(\left|\widehat{\pi}_{0}\right|+\left|\widehat{\pi}_{1}\right|\right)} x^{\nu\left(\widehat{\pi}_{0}\right)-\nu\left(\widehat{\pi}_{1}\right)} \omega^{2\left(\left|\widehat{\pi}_{0}\right|+\left|\widehat{\pi}_{1}\right|\right)} \\
& =\frac{\sum_{k \geq 0} q^{T_{k}}}{\left(x q^{2} \omega^{2}, q^{2} \omega^{2} / x ; q^{2} \omega^{2}\right)_{\infty}}=\frac{\left(q^{4} ; q^{4}\right)_{\infty}\left(-q ; q^{2}\right)_{\infty}}{\left(x q^{2} \omega^{2}, q^{2} \omega^{2} / x ; q^{2} \omega^{2}\right)_{\infty}} .
\end{aligned}
$$

Here we have used (2.22) along with the fact that a partition is a 2-core if and only if it is a staircase 11. Hence using

$$
\left(x^{ \pm 1} q^{2} \omega^{2} ; q^{2} \omega^{2}\right)_{\infty}=\left(x^{ \pm 1} q^{2} \omega^{2}, x^{ \pm 1} q^{4} ; q^{4}\right)_{\infty},
$$

we find that

$$
G_{2}(x, \sqrt{ \pm 1}, q)=\frac{\left(q^{4} ; q^{4}\right)_{\infty}\left(-q ; q^{2}\right)_{\infty}}{\left( \pm x q^{2}, x q^{4}, \pm q^{2} / x, q^{4} / x ; q^{4}\right)_{\infty}} .
$$

Comparing this with the product in Lemma 2.1, we see that

$$
G_{2}(x, \sqrt{ \pm 1}, q)=g(x, \sqrt{ \pm 1}, q) .
$$

This means that

$$
\tilde{p}_{i}(m, n)=p_{i}^{*}(m, n), \quad i=0,2 .
$$

Here $\tilde{p}_{i}(m, n)\left(\right.$ resp. $\left.p_{i}^{*}(m, n)\right)$ denotes the number of partitions of $n$ with srank $\equiv i$ $(\bmod 4)$ and 2 -quotient-rank $=m($ resp. St-crank $=m)$. Theorem 3.1 follows easily from (3.24) and Theorem 2.2 .

We remark that the St-crank and the 2-quotient-rank are distinct statistics. For example, St-crank $((5,4,1))=0$ but 2 -quotient-rank $((5,4,1))=1$. It would be interesting to find a direct combinatorial proof of (3.24).

3.3. 3-cores and the crank. It is natural to attempt to extend the construction of the previous section to (3-core, 3-quotient). To this end we define

$$
G_{3}(x, q):=\sum_{\pi} q^{|\pi|}\left(x^{3 n_{1}}+x^{3 n_{2}+1}+x^{-3 n_{2}-1}\right) x^{3\left(\nu\left(\widehat{\pi}_{1}\right)-\nu\left(\widehat{\pi}_{2}\right)\right)},
$$

where

$$
\begin{aligned}
\phi_{1}(\pi) & =\left(\pi_{3 \text {-core }},\left(\widehat{\pi}_{0}, \widehat{\pi}_{1}, \widehat{\pi}_{2}\right)\right), \\
\phi_{2}\left(\pi_{3 \text {-core }}\right) & =\left(-n_{1}-n_{2}, n_{1}, n_{2}\right) .
\end{aligned}
$$

Hence using (3.3), (3.8) with $t=3$, we find that

$$
|\pi|=Q_{3}\left(n_{1}, n_{2}\right)+3\left(\left|\widehat{\pi}_{0}\right|+\left|\widehat{\pi}_{1}\right|+\left|\widehat{\pi}_{2}\right|\right),
$$

where

$$
Q_{3}\left(n_{1}, n_{2}\right)=3\left(n_{1}^{2}+n_{1} n_{2}+n_{2}^{2}\right)+n_{1}+2 n_{2} .
$$


Clearly,

$$
=\frac{\sum_{n_{1}, n_{2}} q^{Q_{3}\left(n_{1}, n_{2}\right)} x^{3 n_{1}}+\sum_{n_{1}, n_{2}} q^{Q_{3}\left(n_{1}, n_{2}\right)} x^{3 n_{2}+1}+\sum_{n_{1}, n_{2}} q^{Q_{3}\left(n_{1}, n_{2}\right)} x^{-1-3 n_{2}}}{\left(q^{3}, x^{3} q^{3}, q^{3} / x^{3} ; q^{3}\right)_{\infty}} .
$$

Next, we change summation variables in the first, second, and third sums respectively as

$$
\begin{aligned}
3 n_{1} & =n-m, \quad 3 n_{2}=n+2 m, \\
3 n_{1}+1 & =-2 n-m, \quad 3 n_{2}+1=n-m, \\
3 n_{1}+1 & =-n-2 m, \quad 3 n_{2}+1=m-n,
\end{aligned}
$$

respectively. In this way, we get

$$
G_{3}(x, q)=\frac{\sum_{n, m} q^{n^{2}+m n+m^{2}+n+m} x^{n-m}}{\left(q^{3}, x^{3} q^{3}, q^{3} / x^{3} ; q^{3}\right)_{\infty}} .
$$

Remarkably, the numerator on the right side of (3.31) has a product representation. By equation (1.23) in [10] we have

$$
\sum_{n, m} q^{n^{2}+m n+m^{2}+n+m} x^{n-m}=(x+1+1 / x)(q ; q)_{\infty}\left(q^{3} ; q^{3}\right)_{\infty} \frac{\left(x^{3} q^{3} ; q^{3}\right)_{\infty}\left(q^{3} / x^{3} ; q^{3}\right)_{\infty}}{(x q ; q)_{\infty}(q / x ; q)_{\infty}} .
$$

We have

$$
G_{3}(x, q)=(x+1+1 / x) \frac{(q)_{\infty}}{(x q ; q)_{\infty}(q / x ; q)_{\infty}} .
$$

Recalling (1.11), we see that (3.33) gives a surprising relation between 3-cores and the Andrews-Garvan crank. This certainly warrants further investigation.

3.4. 5-cores. We now assume $t=5$. For the case $\delta=4$ the right side of (3.13) can be simplified using the the following change of variables:

$$
\begin{aligned}
& n_{0}=\alpha_{0}+\alpha_{4}, \\
& n_{1}=-\alpha_{0}+\alpha_{1}+\alpha_{4}, \\
& n_{2}=-\alpha_{1}+\alpha_{2}, \\
& n_{3}=-\alpha_{2}+\alpha_{3}-\alpha_{4}, \\
& n_{4}=-\alpha_{3}-\alpha_{4} .
\end{aligned}
$$

We find $\vec{n}$ is an $n$-vector satisfying $\vec{n} \cdot \vec{b}_{5} \equiv 4(\bmod 5)$ if and only if

$$
\vec{\alpha}=\left(\alpha_{0}, \alpha_{1}, \alpha_{2}, \alpha_{3}, \alpha_{4}\right) \in \mathbb{Z}^{5}
$$

and

$$
\alpha_{0}+\alpha_{1}+\alpha_{2}+\alpha_{3}+\alpha_{4}=1 .
$$

We call $\vec{\alpha}$ an $\alpha$-vector. Hence, by (3.13) and (3.14) we have

$$
\sum_{n \geq 0} a_{5}(5 n+4) q^{n+1}=\sum_{\substack{\vec{\alpha} \cdot \overrightarrow{1}_{5}=1 \\ \vec{\alpha} \in \mathbb{Z}^{5}}} q^{Q(\vec{\alpha})}
$$


and

$$
\sum_{n \geq 0} p(5 n+4) q^{n+1}=\frac{1}{(q)_{\infty}^{5}} \sum_{\substack{\vec{\alpha} \cdot \overrightarrow{1}_{5}=1 \\ \vec{\alpha} \in \mathbb{Z}^{5}}} q^{Q(\vec{\alpha})}
$$

where

$$
Q(\vec{\alpha})=\|\vec{\alpha}\|^{2}-\left(\alpha_{0} \alpha_{1}+\alpha_{1} \alpha_{2}+\cdots+\alpha_{4} \alpha_{0}\right),
$$

If $|\pi| \equiv 4(\bmod 5)$ and $t=5$, we can combine bijections $\phi_{1}$ and $\phi_{2}$ into a single bijection

$$
\Phi(\pi)=\left(\vec{\alpha}, \vec{\pi}_{5}\right),
$$

such that

$$
|\pi|=5 Q(\vec{\alpha})-1+5 \sum_{i=0}^{4}\left|\widehat{\pi}_{i}\right|
$$

Next, following [9] we define the 5 -core crank of $\pi$ when $|\pi| \equiv 4(\bmod 5)$ as

$$
c_{5}(\pi)=1+\sum_{i=0}^{4} i \alpha_{i} \equiv 2\left(1+n_{0}-n_{1}-n_{2}+n_{3}\right) \equiv 2+\sum_{i=-2}^{2} i r_{2-i} \quad(\bmod 5)
$$

where $\alpha$ is determined by (3.40).

It is easy to check that $Q(\vec{\alpha})$ in (3.41) remains invariant under the following cyclic permutation

$$
\widehat{C}_{1}(\vec{\alpha})=\left(\alpha_{4}, \alpha_{0}, \alpha_{1}, \alpha_{2}, \alpha_{3}\right),
$$

while $c_{5}(\pi)$ increases by $1(\bmod 5)$ under the map

$$
\widehat{O}(\pi)=\Phi^{-1}\left(\widehat{C}_{1}(\vec{\alpha}), \vec{\pi}_{5}\right) .
$$

In other words, if $|\pi| \equiv 4(\bmod 5)$, then

$$
|\pi|=|\widehat{O}(\pi)|
$$

and

$$
c_{5}(\pi)+1 \equiv c_{5}(\widehat{O}(\pi)) \quad(\bmod 5) .
$$

This suggests that all partitions of $5 n+4$ can be organized into orbits. Each orbit consists of five distinct members:

$$
\pi, \widehat{O}(\pi), \widehat{O}^{2}(\pi), \widehat{O}^{3}(\pi), \widehat{O}^{4}(\pi),
$$

and each element of the orbit has a distinct 5 -core crank $(\bmod 5)$. Clearly, the total number of such orbits is $\frac{1}{5} p(5 n+4)$, and so $p(5 n+4) \equiv 0(\bmod 5)$. This summarizes the combinatorial proof of (1.1) given in 9. If we apply the map $\widehat{O}$ (3.44) to the partitions of $5 n+4$ that are 5 -cores, we find that

$$
a_{5}^{0}(5 n+4)=a_{5}^{1}(5 n+4)=\cdots=a_{5}^{4}(5 n+4),
$$

where, for $0 \leq j \leq 4, a_{5}^{j}(n)$ denotes the number of partitions of $n$ that are 5 -cores with 5 -core crank congruent to $j$ modulo 5 . Hence,

$$
a_{5}^{j}(5 n+4)=\frac{1}{5} a_{5}(5 n+4), \quad j=0,1, \ldots, 4,
$$

which proves that

$$
a_{5}(5 n+4) \equiv 0 \quad(\bmod 5)
$$


Actually, more is true. We have

$$
a_{5}(5 n+4)=5 a_{5}(n) .
$$

We sketch the combinatorial proof of (3.51) given in 9. See also 8. The map $\theta: P_{5 \text {-core }}(n) \longrightarrow P_{5 \text {-core }}^{0}(5 n+4)$, defined in terms of $n$-vectors as

$$
\vec{n} \mapsto \vec{n}^{\prime}=\left(n_{1}+2 n_{2}+2 n_{4}+1,-n_{1}-n_{2}+n_{3}+n_{4}+1,2 n_{1}+n_{2}+2 n_{3},\right.
$$

$$
\left.-2 n_{2}-2 n_{3}-n_{4}-1,-2 n_{1}-n_{3}-2 n_{4}-1\right),
$$

is a bijection. Here $P_{5 \text {-core }}(n)$ is the set of all 5 -cores of $n$, and $P_{5 \text {-core }}^{0}(n)$ is the set of all 5 -cores of $n$ with 5 -core crank congruent to zero modulo 5 . Since $\theta$ is a bijection, we have

$$
a_{5}(n)=a_{5}^{0}(5 n+4) .
$$

The proof of (3.51) follows easily from (3.49) and (3.53). Finally, we remark that Ramanujan's result (1.7) is a straightforward consequence of (3.14) with $(t, \delta)=$ $(5,4)$, (3.51), and (3.5) with $t=5$.

\section{Refinement of Ramanujan's mod 5 congruence, THE SRANK AND THE 5-CORE CRANK}

In the previous section we discussed the combinatorial proof in [9] of Ramanujan's congruence (1.1) using the the 5-core crank (3.42). It is somewhat unexpected that the 5-core crank can be employed to prove the refinement (1.21) as well.

In fact, we were amazed to discover the following elegant formulas:

$$
\begin{aligned}
\operatorname{srank}\left(\pi_{5-\text { core }}\right) \equiv & \sum_{i=0}^{4}\left(n_{i}+i\right)^{3} \quad(\bmod 4), \\
\operatorname{srank}(\pi) \equiv & \operatorname{srank}\left(\pi_{5 \text {-core }}\right)+\sum_{i=0}^{4} \operatorname{srank}\left(\widehat{\pi}_{i}\right) \\
& +2 \sum_{i=0}^{4}\left|\widehat{\pi}_{i}\right|\left(n_{i}+i\right) \quad(\bmod 4),
\end{aligned}
$$

where $\pi_{5 \text {-core }}, \overrightarrow{\hat{\pi}}=\left(\widehat{\pi}_{0}, \widehat{\pi}_{1}, \widehat{\pi}_{2}, \widehat{\pi}_{3}, \widehat{\pi}_{4}\right)$ are determined by (3.1) with $t=5$, and

$$
\vec{n}=\left(n_{0}, n_{1}, \ldots, n_{4}\right)=\phi_{2}\left(\pi_{5 \text {-core }}\right) .
$$

In spite of their simple appearance, the above formulas are far from obvious. In Sections 4 and 5 we prove generalizations of (4.1)-(4.2). Here we restrict our attention to some implications of (4.1) (4.2).

First, we note that if $\left|\pi_{5 \text {-core }}\right| \equiv 4(\bmod 5)$, then (4.1) can be written in terms of an $\alpha$-vector (3.34) as

$\operatorname{srank}\left(\pi_{5 \text {-core }}\right) \equiv \alpha_{0} \alpha_{1}\left(\alpha_{0}-\alpha_{1}\right)+\alpha_{1} \alpha_{2}\left(\alpha_{1}-\alpha_{2}\right)+\cdots+\alpha_{4} \alpha_{0}\left(\alpha_{4}-\alpha_{0}\right) \quad(\bmod 4)$.

Similarly, if $|\pi| \equiv 4(\bmod 5)$, then

$$
\begin{aligned}
\operatorname{srank}(\pi) \equiv & \alpha_{0} \alpha_{1}\left(\alpha_{0}-\alpha_{1}\right)+\cdots+\alpha_{4} \alpha_{0}\left(\alpha_{4}-\alpha_{0}\right)+\sum_{i=0}^{4} \operatorname{srank}\left(\widehat{\pi}_{i}\right) \\
& +2\left\{\left(\alpha_{0}+\alpha_{4}\right)\left|\widehat{\pi}_{0}\right|+\left(\alpha_{2}+\alpha_{3}\right)\left|\widehat{\pi}_{1}\right|+\left(\alpha_{1}+\alpha_{2}\right)\left|\widehat{\pi}_{2}\right|\right. \\
& \left.+\left(\alpha_{0}+\alpha_{1}\right)\left|\widehat{\pi}_{3}\right|+\left(\alpha_{3}+\alpha_{4}\right)\left|\widehat{\pi}_{4}\right|\right\} \quad(\bmod 4) .
\end{aligned}
$$


Remarkably, (4.3) suggests that $\operatorname{srank}\left(\pi_{5 \text {-core }}\right)$ with $\left|\pi_{5 \text {-core }}\right| \equiv 4(\bmod 5)$ remains invariant mod 4 under the cyclic permutation (3.43), and we have the following refinement of (3.49):

$$
a_{5, i}^{j}(5 n+4)=\frac{1}{5} a_{5, i}(5 n+4),
$$

where $j=0, \ldots, 4$ and $i=0,2$. Here $a_{5, i}(n)$ denotes the number of 5 -cores of $n$ with srank $\equiv i(\bmod 4)$, and $a_{5, i}^{j}(n)$ denotes the number of 5 -cores of $n$ with srank $\equiv i(\bmod 4)$ and 5 -core crank $\equiv j(\bmod 5)$. Moreover, it is not difficult to verify that the map $\theta$, given by (3.52), preserves the srank mod 4. Indeed, recalling that $n_{0}+n_{1}+n_{2}+n_{3}+n_{4}=0$ we find after some simplification that

$$
\begin{aligned}
\sum_{i=0}^{4}\left(\left(n_{i}+i\right)^{3}-\left(n_{i}^{\prime}+i\right)^{3}\right) \equiv & 2\left(n_{0} n_{2}\left(n_{0}+n_{2}\right)+n_{1} n_{3}\left(n_{1}+n_{3}\right)+n_{2} n_{3}\left(n_{2}+n_{3}\right)\right. \\
& \left.+n_{1}\left(n_{1}+1\right)+n_{2}\left(n_{2}+1\right)+n_{3}\left(n_{3}+1\right)\right) \\
\equiv & 0 \quad(\bmod 4),
\end{aligned}
$$

where $\vec{n}^{\prime}$ is defined in (3.52). Hence, (3.53) and (3.51) can be refined as

$$
a_{5, i}(n)=a_{5, i}^{0}(5 n+4) \quad(i=0,2),
$$

and

$$
a_{5, i}(5 n+4)=5 a_{5, i}(n) \quad(i=0,2),
$$

respectively.

It is less trivial to prove the 5-core crank analogue of Theorem 2.2. Namely,

Theorem 4.1. The residue of the 5-core crank mod 5 divides the partitions enumerated by $p_{i}(5 n+4)$ with $i=0,2$ into five equal classes.

Proof. We sketch a proof using (4.4) and (4.5). We define the cyclic shift operator $\widehat{C}_{2}$ by

$$
\widehat{C}_{2}\left(\overrightarrow{\widehat{\pi}}_{5}\right)=\left(\widehat{\pi}_{4}, \widehat{\pi}_{2}, \widehat{\pi}_{3}, \widehat{\pi}_{0}, \widehat{\pi}_{1}\right)
$$

Next, we use (4.9) to modify (3.44) as

$$
\widehat{O}_{s}(\pi)=\Phi^{-1}\left(\widehat{C}_{1}(\vec{\alpha}), \widehat{C}_{2}\left(\overrightarrow{\widehat{\pi}}_{5}\right)\right),
$$

where $\Phi(\pi)=\left(\vec{\alpha}, \vec{\pi}_{5}\right)$. Fix $i=0,2$. By (4.4) we see that $\widehat{O}_{s}$ preserves the srank $\bmod 4$, and we may assemble all partitions of $5 n+4$ with srank $\equiv i(\bmod 4)$ into orbits:

$$
\pi, \widehat{O}_{s}(\pi), \widehat{O}_{s}^{2}(\pi), \widehat{O}_{s}^{3}(\pi), \widehat{O}_{s}^{4}(\pi),
$$

where $\pi$ is some partition of $5 n+4$ with $\operatorname{srank}(\pi) \equiv i(\bmod 4)$. As before, each orbit contains exactly five members and the 5 -core crank increases by 1 mod 5 along the orbit. The number of these orbits is $\frac{1}{5} p_{i}(5 n+4)$, consequently $p_{i}(5 n+4) \equiv 0$ $(\bmod 5)$ and the result follows.

Theorem 4.1 is illustrated below in Table 2, which contains all 30 partitions of 9 , organized into 6 orbits. Each row in this table represents an orbit, and the first row lists all partitions of 9 that are 5 -cores. In the table we have also included the image of each partition under the bijection $\phi_{1}$. Instead of giving the full 5 -quotient we have used a short-hand notation. Terms in the table have the form $\pi \rightarrow\left(\pi_{5 \text {-core }}, k\right)$, 
where $k$ indicates that $\vec{\pi}_{5}=\left(\widehat{\pi}_{0}, \widehat{\pi}_{1}, \widehat{\pi}_{2}, \widehat{\pi}_{3}, \widehat{\pi}_{4}\right)$, where $\widehat{\pi}_{i}=(1)$ if $i=k$, and $(0)$ otherwise.

TABLE 2 .

\begin{tabular}{|c|c|c|c|c|c|}
\hline & $c_{5} \equiv 0(\bmod 5)$ & $1(\bmod 5)$ & $2(\bmod 5)$ & $3(\bmod 5)$ & $4(\bmod 5)$ \\
\hline \multirow{4}{*}{$\begin{array}{c}\text { srank } \equiv 0 \\
(\bmod 4)\end{array}$} & $\left(1^{4}, 5^{1}\right)$ & $\left(1^{3}, 3^{2}\right)$ & $\left(1^{4}, 2^{1}, 3^{1}\right)$ & $\left(1^{1}, 2^{1}, 6^{1}\right)$ & $\left(2^{2}, 5^{1}\right)$ \\
\cline { 2 - 6 } & & & & & \\
\cline { 2 - 6 } & $\left(1^{5}, 2^{2}\right) \rightarrow$ & $\left(2^{3}, 3^{1}\right) \rightarrow$ & $\left(1^{2}, 7^{1}\right) \rightarrow$ & $\left(4^{1}, 5^{1}\right) \rightarrow$ & $\left(1^{3}, 2^{1}, 4^{1}\right) \rightarrow$ \\
& $\left(\left(2^{2}\right), 3\right)$ & $\left(\left(1^{4}\right), 2\right)$ & $\left(\left(1^{2}, 2^{1}\right), 1\right)$ & $\left(\left(1^{1}, 3^{1}\right), 4\right)$ & $\left.\left(4^{1}\right), 0\right)$ \\
\cline { 2 - 6 } & $\left(3^{3}\right) \rightarrow$ & $\left(1^{9}\right) \rightarrow$ & $\left(1^{1}, 3^{1}, 5^{1}\right) \rightarrow$ & $\left(1^{2}, 2^{2}, 3^{1}\right) \rightarrow$ & $\left(9^{1}\right) \rightarrow$ \\
& $\left(\left(2^{2}\right), 2\right)$ & $\left(\left(1^{4}\right), 1\right)$ & $\left(\left(1^{2}, 2^{1}\right), 4\right)$ & $\left(\left(1^{1}, 3^{1}\right), 0\right)$ & $\left(\left(4^{1}\right), 3\right)$ \\
\cline { 2 - 6 } & $\left(2^{1}, 7^{1}\right) \rightarrow$ & $\left(1^{2}, 2^{1}, 5^{1}\right) \rightarrow$ & $\left(1^{1}, 2^{4}\right) \rightarrow$ & $\left(1^{6}, 3^{1}\right) \rightarrow$ & $\left(1^{1}, 4^{2}\right) \rightarrow$ \\
& $\left(\left(2^{2}\right), 1\right)$ & $\left(\left(1^{4}\right), 4\right)$ & $\left(\left(1^{2}, 2^{1}\right), 0\right)$ & $\left(\left(1^{1}, 3^{1}\right), 3\right)$ & $\left(\left(4^{1}\right), 2\right)$ \\
\hline \multirow{5}{*}{$\operatorname{srank} \equiv 2$} & $\left(1^{3}, 2^{3}\right) \rightarrow$ & $\left(1^{3}, 6^{1}\right) \rightarrow$ & $\left(2^{1}, 3^{1}, 4^{1}\right) \rightarrow$ & $\left(1^{1}, 8^{1}\right) \rightarrow$ & $\left(1^{2}, 3^{1}, 4^{1}\right) \rightarrow$ \\
& $\left(\left(2^{2}\right), 4\right)$ & $\left(\left(1^{4}\right), 0\right)$ & $\left(\left(1^{2}, 2^{1}\right), 3\right)$ & $\left(\left(1^{1}, 3^{1}\right), 2\right)$ & $\left(\left(4^{1}\right), 1\right)$ \\
\cline { 2 - 6 } & $\left(3^{1}, 6^{1}\right) \rightarrow$ & $\left(1^{1}, 2^{2}, 4^{1}\right) \rightarrow$ & $\left(1^{7}, 2^{1}\right) \rightarrow$ & $\left(1^{1}, 2^{1}, 3^{2}\right) \rightarrow$ & $\left(1^{5}, 4^{1}\right) \rightarrow$ \\
& $\left(\left(2^{2}\right), 0\right)$ & $\left(\left(1^{4}\right), 3\right)$ & $\left(\left(1^{2}, 2^{1}\right), 2\right)$ & $\left(\left(1^{1}, 3^{1}\right), 1\right)$ & $\left(\left(4^{1}\right), 4\right)$ \\
\hline
\end{tabular}

Now, we state some new formulas for $a_{5,0}(n)$ :

$$
\begin{aligned}
a_{5,0}(4 n) & =a_{5}(4 n), \\
a_{5,0}(4 n+1) & =a_{5}(4 n+1), \\
a_{5,0}(4 n+2) & =0, \\
a_{5,0}(4 n+3) & =a_{5}(n) .
\end{aligned}
$$

Formulas (4.11)-(4.13) follow from (4.1). Formula (4.14) is a consequence of the following bijective map, defined in terms of $n$-vectors by

$$
\vec{n} \mapsto \vec{n}^{\prime}=\left(2 n_{1}, 1+2 n_{4}, 2 n_{2},-1+2 n_{0}, 2 n_{3}\right) .
$$

To show that this is a bijection, one may easily verify that

$$
\left|\phi_{2}^{-1}\left(\vec{n}^{\prime}\right)\right|=4\left|\phi_{2}^{-1}(\vec{n})\right|+3,
$$

and show that if $\left|\pi_{5 \text {-core }}\right| \equiv 3(\bmod 4)$, then $\operatorname{srank}\left(\pi_{5 \text {-core }}\right) \equiv 0(\bmod 4)$ if and only if

$$
\phi_{2}\left(\pi_{5 \text {-core }}\right) \equiv(0,1,0,1,0) \quad(\bmod 2) .
$$

\section{THE SRANK OF $t$-CORES}

In this section we generalize (4.1) to $t$-cores for general $t$.

Theorem 5.1. Let $t \geq 2$, and

$$
\phi_{2}\left(\pi_{t-\text { core }}\right)=\vec{n}=\left(n_{0}, n_{1}, n_{2}, \ldots, n_{t-1}\right) .
$$

Let $a=0$ or 1 . If $t \equiv 1+2 a(\bmod 4)$, then

$$
\operatorname{srank}\left(\pi_{t-\text { core }}\right) \equiv \sum_{i=0}^{t-1}\left(n_{i}+(1-2 a) i+a\right)^{3} \quad(\bmod 4),
$$


and if $t \equiv 2 a(\bmod 4)$, then

$$
\operatorname{srank}\left(\pi_{t \text {-core }}\right) \equiv \sum_{i=0}^{t-1} a n_{i}^{2}+\left(i^{2}+i\right) n_{i} \quad(\bmod 4) .
$$

Proof. For a partition $\pi=\left(\lambda_{1}, \lambda_{2}, \ldots, \lambda_{\nu}\right)$,

$$
\operatorname{srank}(\pi) \equiv \sum_{j=1}^{\nu}\left(\lambda_{j}^{2}+(1-2 j) \lambda_{j}\right) \quad(\bmod 4),
$$

by Proposition 3.1(c) in [14]. Now let $t \geq 2$, and suppose $\pi$ is a $t$-core, $\pi=$ $\left(\lambda_{1}, \lambda_{2}, \ldots, \lambda_{\nu}\right)$, and

$$
\phi_{2}(\pi)=\vec{n}=\left(n_{0}, n_{1}, n_{2}, \ldots, n_{t-1}\right) .
$$

Suppose $n_{i}>0$. Then $i$ is exposed in each region $k, 1 \leq k \leq n_{i}$, and this exposed cell is to the right of the main diagonal. Each such exposed $i$ in region $k$ corresponds to a part of the partition in which the number of cells to the right of the main diagonal is $t(k-1)+i$. Thus,

$$
J=\text { size of the Durfee square of } \pi=\sum_{n_{i}>0} n_{i}
$$

and

$$
\begin{gathered}
\operatorname{srank}(\pi) \equiv \sum_{j=1}^{J}\left(\lambda_{j}-j\right)^{2}+\left(\lambda_{j}-j\right)+\left(j-j^{2}\right)+\sum_{j=J+1}^{\nu} \lambda_{j}^{2}+(1-2 j) \lambda_{j} \quad(\bmod 4) \\
\equiv \sum_{n_{i}>0} \sum_{k=1}^{n_{i}}(t(k-1)+i)^{2}+(t(k-1)+i)+\sum_{j=1}^{J} j-j^{2} \\
\quad+\sum_{j=J+1}^{\nu} \lambda_{j}^{2}+(1-2 j) \lambda_{j} \quad(\bmod 4) \\
\equiv \sum_{n_{i}>0} g\left(t, n_{i}, i\right)+\frac{1}{3}\left(J-J^{3}\right)+\sum_{j=J+1}^{\nu} \lambda_{j}^{2}+(1-2 j) \lambda_{j} \quad(\bmod 4),
\end{gathered}
$$

where

$$
g(t, n, i)=\frac{1}{3} t^{2} n^{3}+\left(t i-\frac{1}{2} t(t-1)\right) n^{2}+\left(i^{2}-i(t-1)+\frac{1}{6} t^{2}-\frac{1}{2} t\right) n .
$$

We note that for $n>0$,

$$
g(t, n, i) \equiv 0 \quad(\bmod 2),
$$

since $g(t, n, i)$ is a sum of even integers. A calculation shows that

$$
g(t, n, i)+g(t,-n, t-1-i)=0 .
$$

It follows that $g(t, n, i)$ is a sum of even integers for $n<0$ and (5.7) holds for all $n$, so that

$$
g(t, n, i) \equiv g(t,-n, t-1-i) \quad(\bmod 4) .
$$

We have

$$
\phi_{2}\left(\pi^{\prime}\right)=\left(-n_{t-1},-n_{t-2}, \ldots,-n_{0}\right) .
$$


See [9, p. 3]. Let $\pi_{1}$ be the partition consisting of the first $J$ parts of $\pi^{\prime}$. Then by (5.10), (5.7) and (5.9) we find that

$$
\begin{aligned}
\operatorname{srank}\left(\pi_{1}\right) & \equiv \sum_{n_{i}<0} g\left(t,-n_{i}, t-1-i\right)+\frac{1}{3}\left(J-J^{3}\right) \\
& \equiv \sum_{n_{i}<0} g\left(t, n_{i}, i\right)+\frac{1}{3}\left(J-J^{3}\right) \quad(\bmod 4) .
\end{aligned}
$$

Here we have used the fact that $\pi^{\prime}$ is also a $t$-core. Now,

$$
\operatorname{srank}\left(\pi_{1}^{\prime}\right)=-\operatorname{srank}\left(\pi_{1}\right) \equiv \operatorname{srank}\left(\pi_{1}\right) \quad(\bmod 4)
$$

and

$$
\operatorname{srank}\left(\pi_{1}^{\prime}\right) \equiv \sum_{j=1}^{J} J^{2}+(1-2 j) J+\sum_{j=J+1}^{\nu} \lambda_{j}^{2}+(1-2 j) \lambda_{j} \quad(\bmod 4) .
$$

Hence

$$
\sum_{j=J+1}^{\nu} \lambda_{j}^{2}+(1-2 j) \lambda_{j} \equiv \sum_{n_{i}<0} g\left(t, n_{i}, i\right)+\frac{1}{3}\left(J-J^{3}\right) \quad(\bmod 4)
$$

since

$$
\sum_{j=1}^{J} J^{2}+(1-2 j) J=0
$$

Hence, by (5.5) and (5.14), we have

$$
\begin{aligned}
\operatorname{srank}(\pi) & \equiv \sum_{n_{i}>0} g\left(t, n_{i}, i\right)+\sum_{n_{i}<0} g\left(t, n_{i}, i\right)+\frac{2}{3}\left(J-J^{3}\right) \quad(\bmod 4) \\
& \equiv \sum_{i=0}^{t-1} g\left(t, n_{i}, i\right) \quad(\bmod 4),
\end{aligned}
$$

since $g(t, 0, i)=0$ and $\frac{2}{3}\left(J-J^{3}\right) \equiv 2\left(J^{3}-J\right) \equiv 0(\bmod 4)$.

We prove (5.1) and (5.2) by finding simplified forms for $g(t, n, i)(\bmod 4)$. It is clear that the value of $g(t, n, i)(\bmod 4)$ depends on the residue of $t(\bmod 4)$.

Case $1 . t \equiv 0(\bmod 4)$. Then

$$
g(t, n, i) \equiv g(0, n, i) \equiv\left(i^{2}+i\right) n \quad(\bmod 4) .
$$

Thus (5.2) holds when $a=0$.

Case $2 . t \equiv 1(\bmod 4)$. Then

$$
g(t, n, i) \equiv g(1, n, i) \equiv-g(1, n, i) \equiv(n+i)^{3}-n-i^{3} \quad(\bmod 4),
$$

so that

$$
\begin{aligned}
\operatorname{srank}(\pi) & \equiv \sum_{i=0}^{t-1}\left(n_{i}+i\right)^{3}-\sum_{i=0}^{t-1} n_{i}-\sum_{i=0}^{t-1} i^{3} \quad(\bmod 4) \\
& \equiv \sum_{i=0}^{t-1}\left(n_{i}+i\right)^{3}-\frac{t^{2}(t-1)^{2}}{4}(\bmod 4) \\
& \equiv \sum_{i=0}^{t-1}\left(n_{i}+i\right)^{3} \quad(\bmod 4),
\end{aligned}
$$


$\pi$

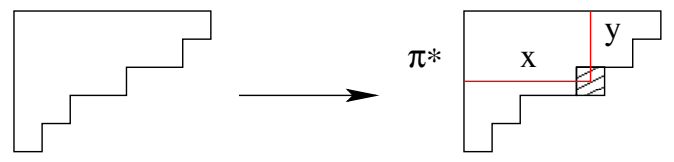

Figure 2. Attaching one cell to the rim

since $t \equiv 1(\bmod 4)$. Here we have also used (3.7). Thus (5.1) holds when $a=0$.

Case $3 . t \equiv 2(\bmod 4)$. Then

$$
\begin{aligned}
g(t, n, i) & \equiv g(2, n, i) \equiv-g(2, n, i) \equiv\left(n^{2}+\left(i^{2}+i\right) n\right)-n+2 i n(n+i) \\
& \equiv\left(n^{2}+\left(i^{2}+i\right) n\right)-n \quad(\bmod 4) .
\end{aligned}
$$

Thus (5.2) holds when $a=1$.

Case $4 . t \equiv 3(\bmod 4)$. Then

$$
g(t, n, i) \equiv g(3, n, i) \equiv-g(3, n, i) \equiv(n-i+1)^{3}+n+(i-1)^{3} \quad(\bmod 4) .
$$

Since

$$
\sum_{i=0}^{t-1}(i-1)^{3}=t \frac{(t-3)}{4}(t(t-3)+4) \equiv 0 \quad(\bmod 4),
$$

for $t \equiv 3(\bmod 4)$, we see that (5.1) holds when $a=1$.

\section{The SRAnK AND The $t$-QUOTIENT}

In this section we prove that

$$
\operatorname{srank}(\pi) \equiv \operatorname{srank}\left(\pi_{t \text {-core }}\right)+2 a \sum_{i=0}^{t-1}\left|\widehat{\pi}_{i}\right| \quad(\bmod 4),
$$

provided $t \equiv 2 a(\bmod 4)$, and

$$
\begin{aligned}
\operatorname{srank}(\pi) \equiv \operatorname{srank}\left(\pi_{t-\text { core }}\right)+2 \sum_{i=0}^{t-1}\left(n_{i}+i+a\right)\left|\widehat{\pi}_{i}\right| \\
+\sum_{i=0}^{t-1} \operatorname{srank}\left(\widehat{\pi}_{i}\right) \quad(\bmod 4)
\end{aligned}
$$

if $t \equiv 1+2 a(\bmod 4)$. Here $a=0,1$ and

$$
\phi_{1}(\pi)=\left(\pi_{t \text {-core }},\left(\widehat{\pi}_{0}, \ldots, \widehat{\pi}_{t-1}\right)\right) .
$$

We note that when $t=5$, equation (6.2) is formula (4.2). To prove (6.1), (6.2) we use (5.3) which we rewrite as

$$
\operatorname{srank}(\pi) \equiv \sum_{j=1}^{\nu}\left(\lambda_{j}^{2}+(2 j-3) \lambda_{j}\right) \quad(\bmod 4),
$$

where $\pi=\left(\lambda_{1}, \lambda_{2}, \ldots, \lambda_{\nu}\right)$.

Next, let $\pi^{*}$ be a partition obtained from $\pi$ by attaching a single cell with coordinates $(x, y)$ to the rim of the diagram of $\pi$ as indicated in Figure 2

It is easy to see that

(6.4) $\operatorname{srank}\left(\pi^{*}\right)-\operatorname{srank}(\pi) \equiv\left(x^{2}-(x-1)^{2}\right)-\left(y^{2}-(y-1)^{2}\right) \equiv 2(x+y) \quad(\bmod 4)$. 
Suppose, we create a new partition $\pi^{* *}$ by attaching a border-strip of length $\ell$ to the diagram of $\pi$, such that the extreme North-East cell (head) of the strip has coordinates $(x, y)$. Repeated use of (6.4) yields the following formula:

$$
\begin{aligned}
& \operatorname{srank}\left(\pi^{* *}\right)-\operatorname{srank}(\pi) \equiv 2(x+y)+2(x+y+1)+\cdots+2(x+y+\ell-1) \\
& \equiv 2 \ell(x+y)+\ell^{2}-\ell \quad(\bmod 4) .
\end{aligned}
$$

Here we have used the fact that a border-strip can be added to the diagram one cell at a time, in such a way that all intermediate diagrams correspond to partitions. It is straightforward to verify that the right side of (6.5) with $\ell=t \lambda$ becomes

$$
2 a \lambda(\bmod 4), \quad \text { if } t \equiv 2 a \quad(\bmod 4),
$$

and

$$
2 a \lambda(x+y+a)+\lambda^{2}-\lambda \quad(\bmod 4), \quad \text { if } t \equiv 1+2 a \quad(\bmod 4),
$$

with $a=0,1$.

Note that (6.6) immediately implies (6.1). To prove (6.2) we need to work a little harder. Let us consider a partition $\tilde{\pi}_{0}$, such that

$$
\phi_{1}\left(\tilde{\pi}_{0}\right)=\left(\pi_{t \text {-core }},\left(\widehat{\pi}_{0}, \ldots, \widehat{\pi}_{i-1},(0), \widehat{\pi}_{i+1}, \ldots, \widehat{\pi}_{t-1}\right)\right) .
$$

This partition has the following property. The rim cells with color $i$ are exposed in all regions $\leq n_{i}$ and are not exposed in all regions $>n_{i}$ of the extended $t$-residue diagram of $\tilde{\pi}_{0}$. This means that the word $W_{i}$ of $\tilde{\pi}_{0}$ has the form

$$
\begin{array}{ccccccc}
\text { Region : } & \cdots \cdots \cdots & n_{i}-1 & n_{i} & n_{i}+1 & n_{i}+2 & \cdots \cdots \cdots \\
W_{i}: & \cdots \cdots \cdots & E & E & N & N & \cdots \cdots \cdots
\end{array}
$$

Let us attach a border-strip of length $\ell=t \lambda$ to $\tilde{\pi}_{0}$ in such a way that the word $W_{i}$ becomes

$$
\begin{array}{cccccccccc}
\text { Region : } & \cdots & n_{i}-\lambda_{1} & n_{i}+1-\lambda_{1} & \cdots & \cdots & n_{i}+1 & n_{i}+2 & n_{i}+3 & \cdots \\
W_{i}: & \cdots & E & N & E & \cdots & E & N & N & \cdots
\end{array}
$$

This way we create a partition $\tilde{\pi}_{1}$ such that

$$
\phi_{1}\left(\widetilde{\pi}_{1}\right)=\left(\pi_{t-\text { core }},\left(\widehat{\pi}_{0}, \ldots, \widehat{\pi}_{i-1},\left(\lambda_{1}\right), \widehat{\pi}_{i+1}, \ldots, \widehat{\pi}_{t-1}\right)\right) .
$$

It is straightforward to verify that for $t$ odd the coordinates $(x, y)$ of the border-strip head satisfy

$$
x+y \equiv n_{i}+i \quad(\bmod 2) .
$$

Hence using (6.7) and (6.8) we find that for $t \equiv 1+2 a(\bmod 4)$

$$
\operatorname{srank}\left(\tilde{\pi}_{1}\right)-\operatorname{srank}\left(\tilde{\pi}_{0}\right) \equiv 2 \lambda_{1}\left(n_{i}+i+a\right)+\lambda_{1}^{2}-\lambda_{1} \quad(\bmod 4),
$$

where $a=0,1$.

Next, we add to the diagram of $\tilde{\pi}_{1}$ a new border-strip of length $t \lambda_{2}$ with $\lambda_{2} \leq \lambda_{1}$, such that $W_{i}$ becomes

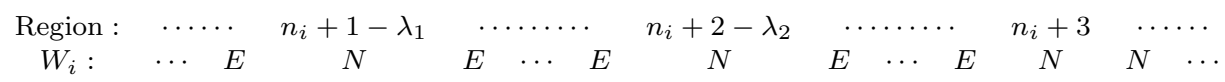

The new partition $\tilde{\pi}_{2}$ satisfies

$$
\phi_{1}\left(\tilde{\pi}_{2}\right)=\left(\pi_{t-\text { core }},\left(\widehat{\pi}_{0}, \ldots, \widehat{\pi}_{i-1},\left(\lambda_{1}, \lambda_{2}\right), \widehat{\pi}_{i+1}, \ldots, \widehat{\pi}_{t-1}\right)\right) .
$$


Replacing $\lambda_{1}$ by $\lambda_{2}$ and $n_{i}$ by $n_{i}+1$ and repeating the argument that led us to (6.9) we obtain

$$
\operatorname{srank}\left(\tilde{\pi}_{2}\right)-\operatorname{srank}\left(\tilde{\pi}_{1}\right) \equiv 2 \lambda_{2}\left(n_{i}+1+i+a\right)+\lambda_{2}^{2}-\lambda_{2} \quad(\bmod 4) .
$$

Let $\tilde{\pi}_{\nu}$ denote the partition obtained from $\tilde{\pi}_{0}$, such that

$$
\phi_{1}\left(\tilde{\pi}_{\nu}\right)=\left(\pi_{t \text {-core }},\left(\widehat{\pi}_{0}, \ldots, \widehat{\pi}_{i}, \ldots, \widehat{\pi}_{t-1}\right)\right),
$$

where $\widehat{\pi}_{i}=\left(\lambda_{1}, \lambda_{2}, \ldots, \lambda_{\nu}\right)$. Proceeding as above and using (6.3) we find that for $t \equiv 1+2 a(\bmod 4)$

$$
\begin{aligned}
\operatorname{srank}\left(\tilde{\pi}_{\nu}\right)-\operatorname{srank}\left(\tilde{\pi}_{0}\right) & \equiv 2 \sum_{j=1}^{\nu} \lambda_{j}\left(n_{i}+i+a+j-1\right)+\sum_{j=1}^{\nu}\left(\lambda_{j}^{2}-\lambda_{j}\right) \\
& \equiv 2\left(n_{i}+i+a\right) \sum_{j=1}^{\nu} \lambda_{j}+\sum_{j=1}^{\nu}\left(\lambda_{j}^{2}+(2 j-3) \lambda_{j}\right) \\
& \equiv 2\left(n_{i}+i+a\right)\left|\widehat{\pi}_{i}\right|+\operatorname{srank}\left(\widehat{\pi}_{i}\right) \quad(\bmod 4),
\end{aligned}
$$

with $a=0,1$. Formula (6.2) follows easily from (6.11).

\section{Generalization of Andrews' refinement AND NEW PARTITION CONGRUENCES MODULO 5}

In Section 3.2 we gave a new combinatorial interpretation of Andrews' result (1.21) in terms of the 2-quotient of a partition. Further study of this development led us to a generalization of (1.21), which we now describe. We define the new partition statistic

$$
\operatorname{BG}-\operatorname{rank}(\pi)=\sum_{j=1}^{\nu}(-1)^{j+1} \operatorname{par}\left(\lambda_{j}\right),
$$

where $\pi=\left(\lambda_{1}, \lambda_{2}, \ldots, \lambda_{\nu}\right)$ and for an integer $m, \operatorname{par}(m)$ denotes the parity of $m$; i.e. $\operatorname{par}(m)=1$ if $m$ is odd and 0 , otherwise. If $\phi_{1}(\pi)=\left(\pi_{2 \text {-core }},\left(\widehat{\pi}_{0}, \widehat{\pi}_{1}\right)\right)$ and $\phi_{2}\left(\pi_{2 \text {-core }}\right)=\left(n_{0},-n_{0}\right)$, then it is easy to verify that

$$
\operatorname{BG}-\operatorname{rank}(\pi)=n_{0}=r_{0}-r_{1} \text {. }
$$

Here $r_{i}$ with $i=0,1$ denotes the number of cells colored $i$ in the 2-residue diagram of $\pi$.

Next, we recall that

$$
\operatorname{srank}(\pi) \equiv|\pi|-\left|\pi_{2 \text {-core }}\right| \equiv|\pi|-n_{0}\left(2 n_{0}-1\right) \quad(\bmod 4) .
$$

Here we have used (3.17) and (3.8). Thus, if $|\pi|$ is given, then $\operatorname{srank}(\pi)(\bmod 4)$ is completely determined by BG-rank $(\pi)$. Clearly, the converse is not true.

Let $\bar{p}_{j}(m, n)$ denote the number of partitions of $n$ with BG-rank $=j$ and 2-quotient-rank $=m$. Then

$$
\begin{aligned}
f_{j}(x, q) & =\sum_{\substack{n \geq 0 \\
m}} \bar{p}_{j}(m, n) x^{m} q^{n} \\
& =q^{(2 j-1) j} \sum_{\widehat{\pi}_{0}, \widehat{\pi}_{1}} q^{2\left(\left|\widehat{\pi}_{0}\right|+\left|\widehat{\pi}_{1}\right|\right)} x^{\nu\left(\widehat{\pi}_{0}\right)-\nu\left(\widehat{\pi}_{1}\right)} \\
& =\frac{q^{(2 j-1) j}}{\left(q^{2} x, q^{2} / x ; q^{2}\right)_{\infty}} .
\end{aligned}
$$


Proceeding as in Section 2 we find that for $\xi^{5}=1, \xi \neq 1$,

$$
f_{j}(\xi, q)=\frac{1}{1-\xi^{2}} \frac{q^{(2 j-1) j}}{\left(q^{10} ; q^{10}\right)_{\infty}} \sum_{n \geq 0}(-1)^{n} q^{n^{2}+n} \xi^{-2 n}\left(1-\xi^{4 n+2}\right) .
$$

We have

$$
f_{j}(\xi, q)=\sum_{\substack{n \geq 0 \\ 0 \leq m \leq 4}} \bar{P}_{j}(m, 5, n) \xi^{m} q^{n}
$$

where $\bar{P}_{j}(m, 5, n)$ denotes the number of partitions of $n$ with BG-rank $=j$ and 2-quotient-rank $\equiv m(\bmod 5)$.

Next, we observe that

$$
\begin{gathered}
n^{2}+n \equiv\left\{\begin{array}{lll}
0 & (\bmod 5), & \text { if } n \equiv 0,4 \quad(\bmod 5), \\
2 & (\bmod 5), & \text { if } n \equiv 1,3 \quad(\bmod 5), \\
1 & (\bmod 5), & \text { if } n \equiv 2 \quad(\bmod 5),
\end{array}\right. \\
(2 j-1) j \equiv\left\{\begin{array}{lll}
0 & (\bmod 5), & \text { if } j \equiv 0,3 \quad(\bmod 5), \\
1 & (\bmod 5), & \text { if } j \equiv 1,2 \quad(\bmod 5), \\
3 & (\bmod 5), & \text { if } j \equiv 4 \quad(\bmod 5),
\end{array}\right.
\end{gathered}
$$

and $\left(1-\xi^{4 n+2}\right)=0$ if $n \equiv 2(\bmod 5)$. This means that

$$
\begin{aligned}
\sum_{m=0}^{4} \bar{P}_{j}(m, 5,5 n) \xi^{m} & =0, \quad \text { if } j \equiv 1,2 \quad(\bmod 5), \\
\sum_{m=0}^{4} \bar{P}_{j}(m, 5,5 n+1) \xi^{m} & =0, \quad \text { if } j \not \equiv 1,2 \quad(\bmod 5), \\
\sum_{m=0}^{4} \bar{P}_{j}(m, 5,5 n+2) \xi^{m} & =0, \quad \text { if } j \not \equiv 0,3 \quad(\bmod 5), \\
\sum_{m=0}^{4} \bar{P}_{j}(m, 5,5 n+3) \xi^{m} & =0, \quad \text { if } j \equiv 0,3 \quad(\bmod 5), \\
\sum_{m=0}^{4} \bar{P}_{j}(m, 5,5 n+4) \xi^{m} & =0, \quad \text { for all } j .
\end{aligned}
$$

Hence we have the following

Theorem 7.1. For $m=0,1,2,3,4$,

$$
\begin{aligned}
\bar{P}_{j}(m, 5,5 n) & =\frac{1}{5} \bar{p}_{j}(5 n), \quad \text { if } j \equiv 1,2 \quad(\bmod 5), \\
\bar{P}_{j}(m, 5,5 n+1) & =\frac{1}{5} \bar{p}_{j}(5 n+1), \quad \text { if } j \neq 1,2 \quad(\bmod 5), \\
\bar{P}_{j}(m, 5,5 n+2) & =\frac{1}{5} \bar{p}_{j}(5 n+2), \quad \text { if } j \neq \equiv 0,3 \quad(\bmod 5), \\
\bar{P}_{j}(m, 5,5 n+3) & =\frac{1}{5} \bar{p}_{j}(5 n+3), \quad \text { if } j \equiv 0,3 \quad(\bmod 5),
\end{aligned}
$$


and

$$
\bar{P}_{j}(m, 5,5 n+4)=\frac{1}{5} \bar{p}_{j}(5 n+4), \quad \text { for all } j .
$$

Here $\bar{p}_{j}(n)$ denotes the number of partitions of $n$ with BG-rank $=j$.

\section{Corollary 7.2.}

$$
\begin{array}{rlll}
\bar{p}_{j}(5 n) \equiv 0 & (\bmod 5) & \text { if } j \equiv 1,2 & (\bmod 5), \\
\bar{p}_{j}(5 n+1) \equiv 0 & (\bmod 5) & \text { if } j \not \equiv 1,2 & (\bmod 5), \\
\bar{p}_{j}(5 n+2) \equiv 0 & (\bmod 5) & \text { if } j \not \equiv 0,3 \quad(\bmod 5), \\
\bar{p}_{j}(5 n+3) \equiv 0 & (\bmod 5) & \text { if } j \equiv 0,3 & (\bmod 5),
\end{array}
$$

and

$$
\bar{p}_{j}(5 n+4) \equiv 0 \quad(\bmod 5) \quad \text { for all } j .
$$

Recalling the comment after (17.3) we see that (7.23) gives an extension of Andrews' result (1.21). However, congruences (7.19)-(7.22) appear to be new. It is possible to modify the construction in Section 4 in order to provide a direct combinatorial proof of (7.23). It is likely that a combinatorial proof of (7.19)-(7.22) would require significant new insights. To make this point plausible we note that the 5 -core analog of (7.19) $-(7.22)$ does not hold. In other words, it is not true that for $r=0,1,2,3$,

$$
\bar{a}_{5, j}(5 n+r) \equiv 0 \quad(\bmod 5)
$$

On the other hand, it can be shown that

$$
\bar{a}_{5, j}(5 n+4) \equiv 0 \quad(\bmod 5)
$$

Here $\bar{a}_{5, j}(n)$ denotes the number of 5-core partitions of $n$ with BG-rank $=j$.

As mentioned before, in [9], $t$-core cranks were given which combinatorially prove all three of Ramanujan's congruences (1.1)-(1.3). It is natural to ask whether the methods of this paper and [9] can be extended to find refinements for Ramanujan's partition congruences mod 7 and 11. In [9], there is a 7-cycle and an 11-cycle analogous to (3.43). However, this 7 -cycle does not preserve the srank $(\bmod 4)$ of 7 -cores of $7 n+5$ so that the srank does not work in this case. One could define a new srank for 7 -cores that is analogous to (4.3); i.e. as a cyclically symmetric polynomial in $\alpha_{0}, \alpha_{1}, \ldots, \alpha_{6}$. The problem is to extend the definition of this statistic to all partitions of $7 n+5$. The authors considered many such polynomials but were unable to identify a simple statistic for partitions of $7 n+5$ which also gave a refinement to Ramanujan's congruence mod 7 analogous to the srank.

It can be shown that the absolute value of the BG-rank of 7 -cores of $7 n+5$ is invariant under the 7-cycle. However, neither the BG-rank nor its absolute value gives a refinement of Ramanujan's congruence mod 7 or 11. And so we would like to pose the

Problem. Is there an analogue of the BG-rank, which gives a refinement of (1.2) and (1.3)?

\section{ACKNOWLEDGEMENTS}

We would like to thank Krishna Alladi, George Andrews, and Alain Lascoux for their interest and comments. 


\section{REFERENCES}

1. G. E. Andrews, On a partition function of Richard Stanley, to appear in the Electronic Journal of Combinatorics volume in honor of Richard Stanley.

2. G. E. Andrews and F. G. Garvan, Dyson's crank of a partition, Bull. Amer. Math. Soc. (N.S.) 18 (1988), 167-171. MR0929094 (89b:11079)

3. A. O. L. Atkin and P. Swinnerton-Dyer, Some properties of partitions, Proc. London Math. Soc. 4 (1954), 84-106. MR0060535 (15:685d)

4. C. E. Boulet, A four-parameter partition identity, preprint.

5. F. J. Dyson, Some guesses in the theory of partitions, Eureka (Cambridge) 8 (1944), 10-15.

6. F. G. Garvan, New combinatorial interpretations of Ramanujan's partition congruences mod 5, 7 and 11, Trans. Amer. Math. Soc. 305 (1988), 47-77. MR0920146 (89b:11081)

7. F. G. Garvan, The crank of partitions mod 8, 9 and 10, Trans. Amer. Math. Soc. 322 (1990), 79-94. MR 1012520 (91b:11109)

8. F. G. Garvan, More cranks and t-cores, Bull. Austral. Math. Soc. 63 (2001), 379-391. MR:1834941 (2002f:11140)

9. F. Garvan, D. Kim and D. Stanton, Cranks and t-cores, Invent. Math. 101 (1990), 1-17. MR.1055707 (91h:11106)

10. M. Hirschhorn, F. Garvan and J. Borwein, Cubic analogues of the Jacobian theta function $\theta(z, q)$, Canad. J. Math. 45 (1993), 673-694. MR1227653 (94m:33011)

11. G. James and A. Kerber, The Representation Theory of the Symmetric Group, AddisonWesley, Reading, MA, 1981. MR0644144 (83k:20003)

12. D. E. Littlewood, Modular representations of symmetric groups, Proc. Roy. Soc. London. Ser. A. 209 (1951), 333-353. MR0049896 (14:243b)

13. A. V. Sills, A combinatorial proof of a partition identity of Andrews and Stanley, preprint.

14. R. P. Stanley, Some remarks on sign-balanced and maj-balanced posets, preprint.

15. A. J. Yee, On partition functions of Andrews and Stanley, preprint.

Department of Mathematics, University of Florida, Gainesville, Florida 32611-8105

E-mail address: alexb@math.ufl.edu

Department of Mathematics, University of Florida, Gainesville, Florida 32611-8105

E-mail address: frank@math.ufl.edu 